\title{
CONSTRUCTION OF INHOMOGENEOUS SYSTEM SOLUTIONS FOR DIFFERENTIAL EQUATIONS IN PARTIAL THIRD-ORDER HYPERGEOMETRIC TYPE
}

\author{
Zhaxylyk Tasmambetov ${ }^{1}$ and Zhanar Ubayeva $^{1}$ \\ ${ }^{1} \mathrm{~K}$ Zhubanov Aktobe Regional State University
}

June 28, 2020

\begin{abstract}
For consideration introduced inhomogeneous near the singularity $(0,0)$ little-studied regular system consisting of two third-order partial differential equations. Distinctive features of constructing a general solution of an inhomogeneous system are installed. A number of specific systems are highlighted from the corresponding generalized homogeneous system of hypergeometric form. For them established a common method for constructing a solution and have been determined the number of linearly independent particular solutions, at the same time regularity conditions are found near the singularity $(0,0)$ and compatibility conditions as well as integrability. The Frobenius-Latysheva method shows the features of constructing general and particular solutions of a homogeneous Clausen system. Constructing a general and particular solutions of nonhomogeneous system Clausen and one partial differential equation of the third order obtained by adding the two equations nonhomogeneous system shown Clausen method of undetermined coefficients.
\end{abstract}

\section{Hosted file}

Tasmambetov_Ubayeva.docx available at https://authorea.com/users/337339/articles/463165construction-of-inhomogeneous-system-solutions-for-differential-equations-in-partialthird-order-hypergeometric-type 